\title{
Cervical lymph node metastases from thyroid cancer: does thyroglobulin and calcitonin measurement in fine needle aspirates improve the diagnostic value of cytology?
}

Enke Baldini ${ }^{1 \dagger}$, Salvatore Sorrenti ${ }^{2 \dagger}$, Cira Di Gioia ${ }^{3}$, Corrado De Vito ${ }^{4}$, Alessandro Antonelli ${ }^{5}$, Lucio Gnessi $^{1}$, Giovanni Carbotta', Eleonora D'Armiento ${ }^{1}$, Paolo Miccoli ${ }^{6}$, Enrico De Antoni ${ }^{2}$ and Salvatore Ulisse ${ }^{1,7^{*}}$

\begin{abstract}
Background: Measurement of thyroglobulin $(\mathrm{Tg})$ protein in the washout of the needle used for fine needle aspiration biopsy cytology (FNAB-C) has been shown to increase the sensitivity of FNAB-C in identifying cervical lymph node (CLN) metastasis from well-differentiated thyroid cancer (TC). In this study, we evaluated whether routine measurement of Tg protein (FNAB-Tgp), Tg mRNA (FNAB-Tgm) and calcitonin (CT) mRNA (FNAB-CTm) in the FNAB washout of CLN increases the accuracy of FNAB-C in the diagnosis of suspicious metastatic CLN.

Methods: In this prospective study 35 CLN from 28 patients were examined. Histology showed metastatic papillary TC (PTC) in 26 CLN, metastatic medullary TC (MTC) in 3 CLN, metastatic anaplastic TC (ATC) in 3 CLN and 3 metastatic CLN from extra-thyroidal cancers.

Results: The overall accuracy of FNAB-C was $84.4 \%$, reaching $95.7 \%$ when the analysis was restricted to PTC. Both FNAB-Tgp and FNAB-Tgm compared favorably with FNAB-C and shown diagnostic performances not statistically different from that of FNAB-C. However, FNAB-Tgp and FNAB-Tgm/FNAB-CTm were found useful in cases in which cytology results were inadequate or provided diagnosis inconsistent with patient's clinical parameters.

Conclusions: We demonstrated that FNAB-C, Tg/CT mRNA and Tg protein determination in the fine-needle washout showed similar accuracy in the diagnosis of metastatic CLN from TC. The results of this study suggest that samples for Tg protein and Tg/CT mRNA measurements from CLN suspicious for metastatic TC should be collected, but their measurements should be restricted to cases in which FNAB-C provides uninformative or inconsistent diagnosis with respect to patient's clinical parameters.
\end{abstract}

Keywords: Thyroid cancer, Lymph node metastasis, Diagnosis, Thyroglobulin, Calcitonin, Fine-needle aspiration cytology, Follow-up

\section{Background}

Thyroid cancer represents the most frequent endocrine neoplasia, accounting for $1.7 \%(0.7 \%$ in male and $2.7 \%$ in female) of all new malignant diseases and $0.5 \%(0.3 \%$ in male and $0.7 \%$ in female) of deaths related to cancer worldwide [1,2]. Thyroid carcinomas originate mostly

\footnotetext{
* Correspondence: salvatore.ulisse@uniroma1.it

${ }^{\dagger}$ Equal contributors

'Department of Experimental Medicine, University of Rome, Rome, Italy

'Department of Experimental Medicine, "Sapienza", University of Rome, Viale Regina Elena, 324, 00161, Rome, Italy

Full list of author information is available at the end of the article
}

from the epithelial follicular cells and are represented for $95 \%$ by the differentiated (DTC) papillary (PTC) and follicular (FTC) carcinomas, while $1-2 \%$ of them are the undifferentiated and unvaryingly fatal anaplastic carcinomas (ATC) [3]. The remaining 3-4\% are medullary thyroid carcinomas (MTC) derived from the parafollicular $\mathrm{C}$ cells [4]. The accurate diagnosis of locoregional lymph node metastasis is of primary importance for the initial surgical approach as well as for prognostic stratification and follow-up [4-9]. In this context, fine-needle aspiration biopsy cytology (FNAB-C) represents the gold

\section{Biomed Central}


standard technique for the detection of cervical lymph node (CLN) metastasis [4-9]. The latter, however, relies on the experience and ability of the cytopathologist, and may be a challenging diagnostic category as CLN could harbor metastasis from a multiplicity of extrathyroidal malignancies or be affected by several non-tumoral diseases [9-11]. In addition, inadequate cellularity or nonrepresentative sampling, often associated with cystic lymph nodes, prevents diagnosis in about $20 \%$ of specimens [12-14]. Over the last two decades, a number of studies have demonstrated that measurement of thyroglobulin protein (Tgp) in the washout of the needle used for FNAB (FNAB-Tgp) increases the sensitivity of FNAB-C in identifying CLN metastasis from DTC [14-27]. As a consequence, routine association of FNAB-Tgp with FNAB-C in the diagnosis of CLN metastasis from papillary and follicular thyroid cancers has been recommended $[6,7,15]$. Similarly, it has been shown that lymph node detection of $\mathrm{Tg}$ mRNA (Tgm) in fineneedle washout implemented the FNAB-C sensitivity for the diagnosis of metastatic CLN from DTC, even if this still needs to be validated on larger case-studies [28].

In the present work we evaluated, in 35 consecutive CLN for which the histological diagnosis was available, if routine measurement of thyroglobulin $(\mathrm{Tg})$ protein and $\mathrm{Tg}$ and calcitonin (CT) mRNA in the washout of the needle used for FNAB increases the sensitivity of FNAB-C in identifying cervical lymph node (CLN) metastasis from either DTC or MTC. The results obtained suggest that while samples for $\mathrm{Tg}$ protein and $\mathrm{Tg} / \mathrm{CT}$ mRNA measurements from CLN suspicious for metastatic thyroid cancer should be always collected, their measurements should be restricted to cases in which FNAB-C gives uninformative or inconsistent diagnosis with respect to patient's biochemical and/or clinical parameters.

\section{Methods \\ Patients}

The case study included 35 cervical lymph nodes (CLN) with definitive diagnosis from 28 consecutive patients referred, from September 2004 to October 2012, to the outpatients' clinic of Endocrinology and Thyroid Diseases of the Policlinico Umberto I general hospital of Rome (Italy). The study was approved by the ethical committee of the Policlinico Umberto I hospital in agreement to the declaration of Helsinki and all patients gave written informed consent. Patients, 7 males and 21 females with a median age of $39.5 \mathrm{yr}$ (range 20-72 yr), with single or multiple suspicious CLN underwent fine-needle aspiration biopsy (FNAB) followed by cytological (FNAB-C) evaluation, Tgp measurement, and Tgm and CTm assessment in the fine needle washout. Among them, 9 patients previously underwent total thyroidectomy with histological diagnosis of papillary thyroid cancer (see Table 1). All patients with thyroid cancer underwent unilateral neck dissection. The histological diagnosis showed metastatic PTC in 21 patients, metastatic MTC in 2, metastatic ATC in 2 and metastasis from extrathyroidal cancers in the remaining 3 patients ( 1 nonHodgkin lymphoma, 1 rhino-pharyngeal and 1 lung carcinoma). The enlarged lymph nodes from the latter 3 patients were evaluated since they all presented thyroid nodules with ultrasound features suspicious of malignancy (i.e. hypoechogenicity, presence of irregular margins and microcalcifications).

\section{Ultrasonography, fine-needle aspiration biopsy (FNAB) and fine-needle washout}

Thyroid ultrasonography (US) was performed on the cervical area using the Aplio XV (Toshiba, Japan) system equipped with a linear transducer (PLT-805AT). The scanning was performed on patients' neck hyperextended in supine position. US parameters suggestive of malignant lymph node infiltration included: rounded shape and long-to-short axis ratio inferior to 1.5; irregular echogenicity; presence of microcalcifications; irregular vascularity, i.e. peripheral or mixed (hilar and peripheral) vascularity. Following US, patients selected for the FNAB were instructed not to take aspirin or any other anticoagulant in the 5 days prior to biopsy. A 25 -gauge needle, attached to $20 \mathrm{ml}$ plastic syringe, was used to aspirate nodes under US assistance. Two to three separate aspiration from each CLN were made. All aspirates were smeared directly on glass slides for cytological examination as described below. The needle was then washed with $1 \mathrm{ml}$ of phosphate buffered saline (PBS) by multiple pumping actions and the suspension was centrifuged at $1200 \mathrm{rpm}$ for $5 \mathrm{~min}$. The supernatant was collected and frozen to $-20^{\circ} \mathrm{C}$ until analyzed for $\mathrm{Tg}$ protein level (FNAB-Tgp), while the pellet was quickly frozen in liquid nitrogen and stored at $-80^{\circ} \mathrm{C}$.

\section{Cytological and histological analysis}

Following FNAB the needle's material was expelled onto glass slides and smeared with a second slide to spread the material across the surface. The slides were then either air-dried or wet-fixed using the Bio-Fix (BioOptica, Milan, Italy). Air-dried slides were stained with a May Grunwald-Giemsa solution, while the wet-fixed slides were stained with the Papanicolaou solution. For the histological diagnosis, soon after lymphadenectomy the tissue was placed in $10 \%$ buffered formalin and paraffin embedded. Four $\mu \mathrm{m}$ thick sections were then prepared and stained with hematoxylin and eosin [29]. Both cytological and histological diagnoses were made by two expert pathologists and were concordant in all cases. Both pathologists were, at the time of their diagnoses, 
Table 1 Cytological, molecular and histological diagnoses of suspicious metastatic cervical lymph nodes (CLN) from thyroid cancer patients

\begin{tabular}{|c|c|c|c|c|c|}
\hline CLN number & FNAB-C & FNAB-Tgp (ng/FNAB) & FNAB-Tgm & FNAB-CTm & Histology \\
\hline 1 & IN & 6620 & + & - & Met. PTC \\
\hline 2 & IN & 7480 & + & - & Met. PTC \\
\hline 3 & Met. PTC & 5652 & + & - & Met. PTC \\
\hline $4^{*}$ & Met. PTC & 5293 & + & - & Met. PTC \\
\hline $5^{*}$ & Met. PTC & 18612 & + & - & Met. PTC \\
\hline 6 & Met. PTC & 1625 & + & - & Met. PTC \\
\hline 7 & Met. MTC & 0.9 & - & + & Met. MTC \\
\hline $8^{*}$ & IN & 37.50 & IN & IN & Met. PTC \\
\hline 9 & Met. PTC & 26108 & + & - & Met. PTC \\
\hline 10 & Met. ATC & UND & + & - & Met. ATC \\
\hline 11 & Met. PTC & 37250 & ND & ND & Met. PTC \\
\hline 12 & Met. PTC & 0.8 & + & - & Met. ATC \\
\hline 13 & Met. PTC & 2.82 & - & - & Met. ATC \\
\hline $14^{*}$ & Met. PTC & 114 & + & - & Met. PTC \\
\hline $15^{*}$ & Met. PTC & 288 & - & - & Met. PTC \\
\hline 16 & Met. PTC & 1151 & + & - & Met. PTC \\
\hline 17 & Met. PTC & 3714 & + & - & Met. PTC \\
\hline 18 & Met. PTC & 480 & + & - & Met. PTC \\
\hline 19 & Met. PTC & UND & - & + & Met. MTC \\
\hline 20 & Met. PTC & UND & - & + & Met. MTC \\
\hline $21 *$ & Met. PTC & 62908 & IN & IN & Met. PTC \\
\hline 22 & Met. PTC & 72.3 & + & - & Met. PTC \\
\hline 23 & Met. PTC & 10349 & + & - & Met. PTC \\
\hline 24 & Met. PTC & 384 & ND & ND & Met. PTC \\
\hline $25^{*}$ & Met. PTC & 728 & ND & ND & Met. PTC \\
\hline 26 & Met. PTC & 53 & + & - & Met. PTC \\
\hline 27 & Met. PTC & ND & + & - & Met. PTC \\
\hline $28^{*}$ & Met. PTC & ND & + & - & Met. PTC \\
\hline $29 *$ & Met. PTC & ND & + & - & Met. PTC \\
\hline 30 & Epithelial CA & UND & - & - & Met. RFC \\
\hline 31 & Lymphoma & 10.9 & - & - & Lymphoma \\
\hline 32 & Met. LC & UND & - & - & Met. LC \\
\hline 33 & Met. PTC & 3361 & + & - & Met. PTC \\
\hline $34^{*}$ & Benign & 4395 & - & - & Met. PTC \\
\hline $35^{*}$ & Met. PTC & 4388 & + & - & Met. PTC \\
\hline
\end{tabular}

FNAB-C, fine-needle aspiration biopsy (FNAB) cytology; FNAB-Tgp, FNAB thyroglobulin protein; FNAB-Tgm, FNAB Tg mRNA; FNAB-CTm, FNAB CT mRNA; ND, nondetermined; UND, undetectable; IN, inadequate; Pos. WBS, positive whole body scan; RFC, rhino-pharyngeal carcinoma; CA, cancer; LC, lung cancer. ${ }^{*} C L N$ from previously thyroidectomized patients.

unaware of Tgp, Tgm and CTm results as well the molecular biologists were unaware, at the time of their diagnoses, of the patologists diagnoses.

\section{Tg protein and $\mathrm{Tg}$ autoantibody measurements}

Tgp levels in the FNAB washout were measured using the immunoluminometric assay (ILMA) Tg-PluS (B.R.A.H.M.S.,
Hennigsdorf, Germany). The analytical and functional sensitivity of the assay was, respectively, $0.02 \mathrm{ng} / \mathrm{ml}$ and $0.15 \mathrm{ng} / \mathrm{ml}$. Tg values below the functional sensitivity were considered undetectable [30]. Samples were assayed in duplicate either non-diluted or following 1:10, 1:100 and 1:1000 dilutions in PBS. Results were expressed as ng/FNAB [19]. Even if FNAB-Tgp measurement has 
been reported to be non-affected by serum $\mathrm{Tg}$ autoantibodies [31], all FNAB washout samples were routinely assayed for Tg autoantibodies using the Anti-Tgn kit from B.R.A.H.M.S. with a functional sensitivity of $20 \mathrm{U} / \mathrm{ml}$. In all samples $\mathrm{Tg}$ autoantibodies were undetectable (below $20 \mathrm{U} / \mathrm{ml}$ ).

\section{Extraction and analysis of RNA}

The above mentioned pellets have been resuspended in $500 \mu \mathrm{l}$ of Isol-RNA lysis reagent (Eppendorf, Milano, Italy) and total RNA extracted as previously described [32]. In particular, the precipitation of RNA has been maximized by adding $20 \mu \mathrm{g}$ of molecular biology-grade glycogen to isopropanol. The RNA has been reversetranscribed using anchored Oligo(dT)23 primers and M-MLV reverse transcriptase (Sigma Aldrich Co. St-Louis, MO). Negative controls have been performed in parallel for all samples omitting the reverse transcriptase in the RT reaction mix. PCR mix has been prepared with dNTPs $0.2 \mathrm{mM}, 1.5 \mathrm{U}$ of HotMaster Taq DNA Polymerase (Eppendorf, Milano, Italy), $5 \mu \mathrm{l}$ of 10x Taq buffer containing $\mathrm{Mg}^{2+}$, specific $\mathrm{Tg}, \mathrm{CT}$ and beta-2-microglobulin (B2M) primers $0.5 \mu \mathrm{M}, 2-3 \mu \mathrm{l}$ of cDNA, and molecular biologygrade water to $50 \mu \mathrm{l}$. In particular, the sequences of the above primers were as follows: $\mathrm{Tg}$, forward CTCTGGAAA GATTCTGACATGG (exon 28-29), reverse CTCTGGAA AGATTCTGACATGG (exon 30-31) (amplicon 243 bp); CT, forward CCTTCCTGGCTCTCAGCATC (exon 2), reverse GAGTTTAGTTGGCATTCTGG (exon 4) (amplicon 407); B2M, forward CAGCAGAGAATGGAAAGTC (exon 2), reverse CATGCTGCTTACATGTCTCG (exon 3) (amplicon $269 \mathrm{bp}$ ). After 2 min of initial denaturation at $94^{\circ} \mathrm{C}, 40$ cycles have been run as follows: $94^{\circ} \mathrm{C}$ for $20 \mathrm{sec}, 56-60^{\circ} \mathrm{C}$ for $10 \mathrm{sec}, 65^{\circ} \mathrm{C}$ for $50 \mathrm{sec}$. Positive controls, represented by normal human thyroid tissue for Tg mRNA and the MTC derived cell line TT for CT mRNA, have been included each time. The obtained amplicons have been visualized by agarose gel electrophoresis, and their specificities have been checked by automated DNA sequencing (Primm, Milano, Italy).

\section{Statistical analysis}

Sensitivity, specificity, diagnostic accuracy, positive and negative predictive value of each diagnostic test were calculated. Differences in sensitivity, specificity, negative and positive predictive values and accuracy among the different tests were evaluated by the Fisher exact test. Cohen's k coefficient was used to estimate the agreement between various diagnostic tests. Statistical calculations were performed using Stata version 8.0 (College Station, Texas, Stata Corporation, 2003). The results were considered statistically significant when the two-tailed $\mathrm{p}$ value was $<0.05$.

\section{Results}

FNAB cytology (FNAB-C) versus histological diagnosis

As reported in Table 1, FNAB-C was inadequate in 3 (8.6\%) out the 35 samples of CLN, 2 of which were from anaechoic (cystic) CLN and 1 from hypoechoic CLN. The FNAB-C diagnosis of the 32 CLN was correctly provided in 27 cases, with accuracy of $84.4 \%$. In the remaining 5 cases FNAB-C diagnosis of metastatic PTC in 4 CLN was incorrect because the histology demonstrated 2 metastatic MTC (Table 1, CLN n. 19 and 20) and 2 metastatic ATC (Table 1, CLN n. 12 and 13), while the fifth CLN with a benign cytology turned out to be a metastatic PTC (Table 1, CLN n. 34) These 5 FNAB-C diagnoses were considered as false negative. The specificity, sensitivity, positive (PPV) and negative (NPV) predictive values and accuracy of FNAB-C are reported in Table 2.

FNAB Tg protein (FNAB-Tgp) versus histological diagnosis As reported in Figure 1 and Table 1, Tgp level in the fine-needle washout was measured in 32 out of the 35 CLN with definitive diagnosis. In order to limit false negative results considered unacceptable and in agreement with other studies, we decided to adopt a Tgp cut-off value of $1 \mathrm{ng} / \mathrm{FNAB}$ [16]. FNAB-Tgp provided correct diagnoses in 29 CLN (24 true positive and 5 true negative), false negative results (Tgp level $<1 \mathrm{ng} / \mathrm{FNAB}$ ) in 2 harboring metastatic ATC and false positive result in one with definitive diagnosis of lymphoma. Among the 23 CLN in which histology showed metastatic PTC, Tgp values ranged from $37.5 \mathrm{ng}$ to $62908 \mathrm{ng}$ (mean 8742 ng/FNAB; SD 15001 ng). In Table 2, specificity, sensitivity, PPV, NPV and accuracy of FNAB-Tgp are reported.

\section{FNAB Tg (FNAB-Tgm) and CT (FNAB-CTm) mRNA versus histological diagnosis}

$\mathrm{Tg}$ and CT mRNA levels were measured in 32 out of the 35 CLN with definitive diagnosis. In 2 cases samples were judged inadequate because of the absence of $\beta 2$ microglobulin mRNA. As shown in Tables 1 and 2, the analysis of $\mathrm{Tg}$ mRNA in the fine-needle washout of the 30 samples provided a correct diagnosis in 27 cases (21 true positive and 6 true negative CLN) and 3 false negative results, 1 with definitive diagnosis of metastatic ATC and 2 of metastatic PTC.

The CT mRNA analysis provided correct results in all 30 samples ( 3 true positive and 27 true negative).

\section{Comparison of the different tests in the diagnosis of suspicious CLN}

The comparison of the diagnostic parameters of FNABC, FNAB-Tgp and FNAB-Tgm, alone or in combination, was performed excluding from the case series 3 CLN harboring metastasis from MTC and 3 CLN affected by 
Table 2 Diagnostic performance of different tests for diagnosis of suspicious metastatic cervical lymph nodes (CLN) from thyroid cancer patients $(n=35)$

\begin{tabular}{lccccc}
\hline Test & Sensitivity & Specificity & PPV & NPV & Accuracy \\
\hline FNAB-C & $84.4(27 / 32)[67.2-94.7]$ & N.D. (0/0) [N.D.] & $100(27 / 27)[87.2-100]$ & N.D. (0/5) [0-52.2] & $84.4(27 / 32)[67.2-94.7]$ \\
FNAB-Tgp & $92.3(24 / 26)[74.9-99.1]$ & $83.3(5 / 6)[35.9-99.6]$ & $96.0(24 / 25)[79.7-99.9]$ & $71.4(5 / 7)[29.0-96.3]$ & $90.6(29 / 32)[77.7-103.5]$ \\
FNAB-Tgm & $87.5(21 / 24)[67.6-97.3]$ & $100(6 / 6)[54.1-100]$ & $100(21 / 21)[83.9-100]$ & $66.7(6 / 9)[29.9-92.5]$ & $90.0(27 / 30)[76.3-103.7]$ \\
FNAB-CTm & $100(3 / 3)[29.2-100]$ & $100(27 / 27)[87.2-100]$ & $100(3 / 3)[29.2-100]$ & $100(27 / 27)[87.2-100]$ & $100(30 / 30)[100-100]$ \\
\hline
\end{tabular}

PPV, positive predictive value; NPV, negative predictive value; C, cytology; Tgp, thyroglobulin protein; Tgm, thyroglobulin mRNA; CTm, calcitonin mRNA. N.D., nondetermined. In round brackets the number of cases. In square brackets the $95 \%$ confidence interval.

extrathyroidal cancers. Specificity, sensitivity, PPV, NPV and accuracy are reported in Table 3.

As it may be noticed, the diagnostic performances were comparable and not significantly different among FNAB-Tgp, FNAB-Tgm and FNAB-C. In addition, the combined use of FNAB-Tgp + FNAB-Tgm or FNAB-C + FNAB-Tgp + FNAB-Tgm did not improve significantly the diagnostic value of FNAB-C alone (Table 3).

When the analysis was restricted to the 26 CLN harboring metastatic PTC FNAB-C and FNAB-Tgp showed, respectively, 95.7 and 100\% accuracy and, in the 20 CLN in which both diagnoses were available, a 95\% overall agreement between the 2 tests was observed (Table 3). On the other hand, FNAB-Tgm showed $90.5 \%$ accuracy and an overall agreement of 94.7\% (Cohen's $\mathrm{k}=0.64, \mathrm{p}<0.01$ ) with FNAB-C in the $19 \mathrm{CLN}$ in which both diagnoses were attained.

Regarding the 3 CLN harboring metastatic ATC (Table 1, CLN number 10, 12 and 13), FNAB-C identified correctly one of them (CLN number 10) and gave the erroneous

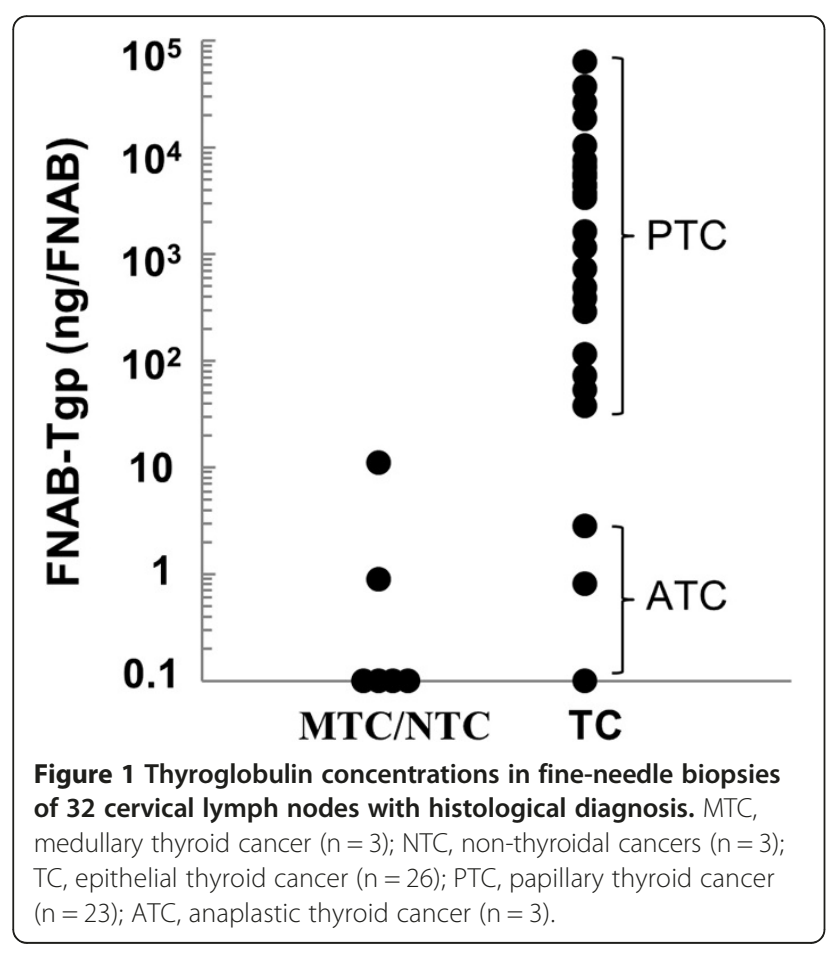

diagnosis of metastatic PTC in the remaining two (from the same patient). As expected, in these 3 CLN FNAB-Tgp levels were low, only one (Table 1, CLN number 13) being above the cut-off value. On the other hand, FNAB-Tgm was positive in two CLN (Table 1, CLN number 10 and 12) and negative in one.

FNAB-Tgp provided a correct diagnosis in all 3 CLN with uninformative cytology. Also FNAB-Tgm provided correct diagnosis in 2 of the 3 CLN with inadequate cytology, being inadequate as well as in the third case.

\section{Discussion}

Cytological evaluation of FNAB represents the gold standard technique for the diagnosis of CLN suspected to harbor metastatic disease from thyroid cancer as well as from other primary tumors. The technique accuracy, highly dependent on the experience and ability of the cytopathologist, has been reported to vary from $73 \%$ to $94 \%[17,18,22,25]$. Over the last years, following clinical evidence showing that FNAB-Tgp in fine-needle washout improves the accuracy of FNAB-C in the evaluation of CLN metastases of DTC, it has been recommended the routine association of FNAB-Tgp with FNAB-C in the preoperative diagnosis of suspicious CLN [6,7,14-27,33]. We here report our experience with $35 \mathrm{CLN}$ for which a definitive diagnosis was available, including cases harboring metastases from PTC, MTC, ATC and other primary tumors.

The results showed that, on 32 CLN harboring metastases from papillary and anaplastic thyroid carcinomas, FNAB-C had $88.5 \%$ accuracy, that is within the range reported in other studies $[17,18,22,25]$. When FNAB-Tgp and FNAB-Tgm were used singly they had a diagnostic performance comparable and not statistically different from that of FNAB-C. In addition, the combination of FNAB-Tgp and/or FNAB-Tgm with FNAB-C did not improve significantly the diagnostic value of FNAB-C. On the other hand, both FNAB-Tgp and FNAB-Tgm compared favorably with FNAB-C. As expected, a major disagreement among the three diagnostic tests was observed in the 3 CLN harboring metastasis from ATC (Table 2, CLN number 10, 12 and 13). It is worth to note that although ATC are undifferentiated cancers, i.e. devoid of thyroid specific gene expression, we consider 
Table 3 Diagnostic performance of the different tests employed in the diagnosis of suspicious metastatic cervical lymph nodes (CLN) from thyroid cancer patients

\begin{tabular}{|c|c|c|c|c|c|}
\hline Test & Sensitivity & Specificity & PPV & NPV & Accuracy \\
\hline \multicolumn{6}{|l|}{$P T C+A T C C L N(n=29)$} \\
\hline FNAB-C & $88.5(23 / 26)$ [69.9-97.6] & N.D. (0/0) [N.D.] & $100(23 / 23)[85.2-100]$ & N.D. (0/3) [0-70.8] & $88.5(23 / 26)$ [69.9-97.6] \\
\hline FNAB-Tgp & $92.3(24 / 26)$ [74.9-99.1] & N.D. (0/0) [N.D.] & $100(24 / 24)[85.8-100]$ & N.D. (0/2) [0-84.2] & $92.3(24 / 26)$ [74.9-99.1] \\
\hline FNAB-Tgm & $87.5(21 / 24)$ [88.1-100] & N.D. (0/0) [N.D.] & $100(21 / 21)$ [84.0-100] & N.D. (0/3) [0-70.8] & $87.5(21 / 24)$ [88.1-100] \\
\hline FNAB-Tgp +- Tgm & $100(29 / 29)$ [88.1-100] & N.D. (0/0) [N.D.] & $100(29 / 29)$ [88.1-100] & N.D. (O/0) [N.D.] & 100 (29/29) [88.1-100] \\
\hline FNAB-C +- Tgp & 96.6 (28/29) [82.2-99.9] & N.D. (0/0) [N.D.] & $100(28 / 28)$ [87.7-100] & N.D. (0/1) [0-97.5] & 96.6 (28/29) [82.2-99.9] \\
\hline FNAB-C + -Tgm & $92.9(26 / 28)$ [76.5-99.1] & N.D. (0/0) [N.D.] & $100(26 / 26)[86.8-100]$ & N.D. (0/2) [0-84.2] & $92.9(26 / 28)$ [76.5-99.1] \\
\hline FNAB-C +-Tgp +- Tgm & 100 (29/29) [88.1-100] & N.D. (0/0) [N.D.] & $100(29 / 29)$ [88.1-100] & N.D. (0/0) [N.D.] & 100 (29/29) [88.1-100] \\
\hline \multicolumn{6}{|l|}{ Only PTC CLN $(n=26)$} \\
\hline FNAB-C & $95.7(22 / 23)$ [78.1-99.9] & N.D. (0/0) [N.D.] & $100(22 / 22)$ [84.6-100] & N.D. (0/1) [0-97.5] & $95.7(22 / 23)$ [78.1-99.9] \\
\hline FNAB-Tgp & 100 (23/23) [85.2-100] & N.D. (0/0) [N.D.] & $100(23 / 23)$ [85.2-100] & N.D. (0/0) [N.D.] & $100(23 / 23)$ [85.2-100] \\
\hline FNAB-Tgm & $90.5(19 / 21)$ [69.6-98.8] & N.D. $(0 / 0)$ [N.D.] & $100(19 / 19)$ [82.4-100] & N.D. (0/2) [0-84.2] & $90.5(19 / 21)$ [69.6-98.8] \\
\hline
\end{tabular}

Upper panel includes 26 CLN with metastatic PTC and ATC. Lower panel includes 23 CLN with only metastatic PTC. PPV, positive predictive value; NPV, negative predictive value; C, cytology; Tgp, thyroglobulin protein; Tgm, thyroglobulin mRNA. In bracket the number of cases. N.D., non-determined.

the absence or the low level (below the cut-off value of $1 \mathrm{ng} / \mathrm{FNAB}$ ) of Tgp or the absence of Tgm as false negative results. In fact, in one of the three metastatic CLN from ATC the detectable Tgp correctly diagnosed one of them (CLN number 13), and the detectable Tgm was diagnostic in the other two (CLN number 10 and 12).

A major diagnostic value of FNAB-Tgp and FNABTgm was observed in cases of uninformative FNAB-C, given that in all 3 CLN characterized by inadequate cytology FNAB-Tgp provided the correct diagnosis. In these cases, FNAB-Tgm also provided the correct diagnosis in 2 of them, being inadequate in one. Other instances in which the combined use of FNAB-Tgp, FNAB-Tgm and FNAB-CTm is valuable include those cases in which FNAB-C diagnosis is not consistent with patient's clinical and biochemical parameters. This was the case (Table 1, CLN number 19 and 20) of the patient harboring metastatic MTC and characterized by elevated level of serum calcitonin in which FNAB-C indicated metastatic PTC, while on the contrary both FNAB-Tgp and FNAB-Tgm were negative, and FNAB-CTm positive.

\section{Conclusions}

FNAB-C remains the gold standard technique for the diagnosis of suspicious CLN due to its ability to efficiently diagnose metastasis not only from thyroid cancers, but also from other primary tumors. If confirmed on larger case studies, the results of this study suggest that samples

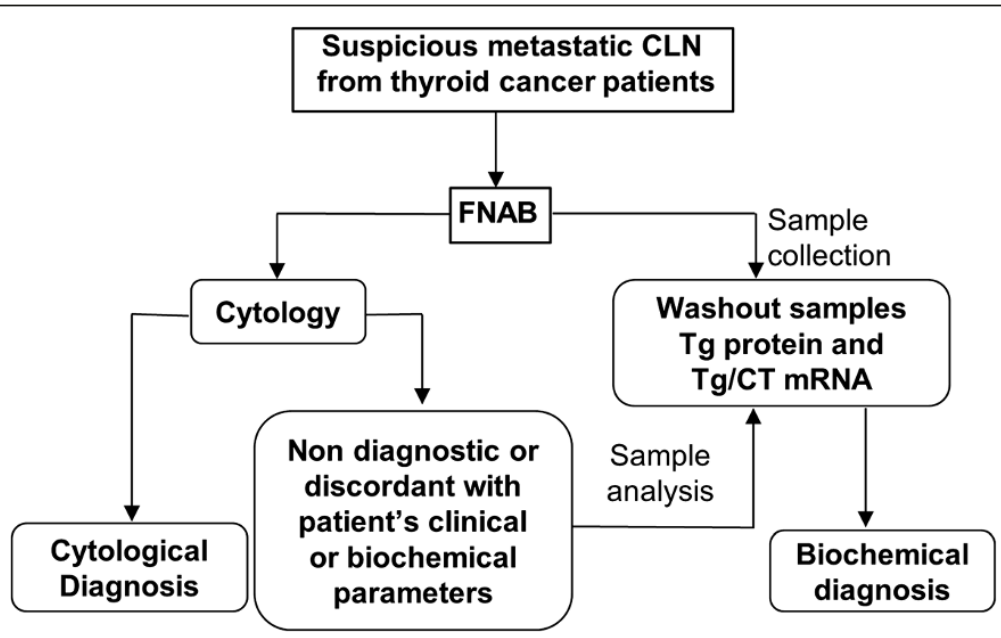

Figure 2 Flow-chart for diagnosis of suspicious metastatic cervical lymph nodes (CLN) from thyroid cancer patients. Following fineneedle aspiration biopsies (FNAB) samples for $\mathrm{Tg}$ protein and $\mathrm{Tg} / \mathrm{CT}$ mRNA measurements should be collected but their analysis restricted to cases with uninformative or clinically unsound FNAB-C diagnosis. Tg, thyroglobulin; CT, calcitonin. 
for $\mathrm{Tg} / \mathrm{CT}$ mRNA and $\mathrm{Tg}$ protein measurements from CLN suspicious for metastatic thyroid cancer should be always collected (Figure 2), but their measurements should be restricted to cases in which FNAB-C give uninformative or inconsistent diagnosis with respect to patient's biochemical and/or clinical parameters. Finally, it is also worth to mention that this approach may significantly reduce the costs of the management of patients with thyroid cancer whose incidence has been increasing over the last years, being actually the fifth most common cancer in women.

\section{Competing interests}

The authors declare that they have no competing interests.

\section{Authors' contribution}

$E B, S S, P M, E D e A$ and SU conceived the study. EB, CDG, AA, LG, GC and EDA run the experimental procedures and organized data collection. SU and CDV performed the statistical analysis. SS, PM, EDeA and SU drafted the manuscript. All authors read and approved the final manuscript.

\section{Acknowledgment}

The authors are very grateful to Dr. Renzo Mocini for the English revision.

\section{Author details}

'Department of Experimental Medicine, University of Rome, Rome, Italy. ${ }^{2}$ Department of Surgical Sciences, University of Rome, Rome, Italy. ${ }^{3}$ Department of Radiological, Oncological and Anato-Pathological Sciences, University of Rome, Rome, Italy. ${ }^{4}$ Department of Public Health and Infectious Diseases, "Sapienza" University of Rome, Rome, Italy. ${ }^{5}$ Department of Interna Medicine, University of Pisa, Pisa, Italy. ${ }^{6}$ Department of Surgery, University of Pisa, Pisa, Italy. ${ }^{7}$ Department of Experimental Medicine, "Sapienza", University of Rome, Viale Regina Elena, 324, 00161, Rome, Italy.

Received: 18 December 2012 Accepted: 11 February 2013

Published: 19 February 2013

\section{References}

1. Ferlay J, Shin HR, Bray F, Forman D, Mathers C, Parkin DM: Estimates of worldwide burden of cancer in 2008: GLOBOCAN 2008. Int J Cancer 2010, 127:2893-2917.

2. Trimboli P, Ulisse S, Graziano FM, Marzullo A, Ruggieri M, Calvanese A, Piccirilli F, Cavaliere R, Fumarola A, D'Armiento M: Trend in thyroid carcinoma size, age at diagnosis and histology in a retrospective study of 500 cases diagnosed over 20 years. Thyroid 2006, 16:1151-1155.

3. Sherman SI: Thyroid carcinoma. Lancet 2003, 36:501-511.

4. American Thyroid Association Guidelines Task Force, Kloos RT, Eng C, Evans $\mathrm{DB}$, Francis GL, Gagel RF, Gharib H, Moley JF, Pacini F, Ringel MD, Schlumberger M, Wells SA Jr: Medullary thyroid cancer: management guidelines of the American Thyroid Association. Thyroid 2009, 19:565-612.

5. American Thyroid Association (ATA) Guidelines Taskforce on Thyroid Nodules and Differentiated Thyroid Cancer, Cooper DS, Doherty GM Haugen BR, Kloos RT, Lee SL, Mandel SJ, Mazzaferri EL, Mclver B, Pacini F. Schlumberger M, Sherman SI, Steward DL, Tuttle RM: Revised American Thyroid Association management guidelines for patients with thyroid nodules and differentiated thyroid cancer. Thyroid 2009, 19:1167-1214.

6. Schlumberger M, Berg G, Cohen O, Duntas L, Jamar F, Jarzab B, Limbert E, Lind P, Pacini F, Reiners C, Sánchez Franco F, Toft A, Wiersinga WM: Followup of low-risk patients with differentiated thyroid carcinoma: a European perspective. Eur J Endocrinol 2004, 150:105-112.

7. Pacini F, Schlumberger M, Dralle H, Elisei R, Smit JW, Wiersinga W: European Thyroid Cancer Taskforce: European consensus for the management of patients with differentiated thyroid carcinoma of the follicular epithelium. Eur J Endocrinol 2006, 154:787-803.

8. Cervin JR, Silverman JF, Loggie BW, Geisinger KR: Virchow's node revisited. Analysis with clinicopathologic correlation of 152 fine-needle aspiration biopsies of supraclavicular lymph nodes. Arch Pathol Lab Med 1995, 8:727-730.
9. Stack BC Jr, Ferris RL, Goldenberg D, Haymart M, Shaha A, Sheth S, Sosa JA, Tufano RP: American Thyroid Association Surgical Affairs Committee: American thyroid association consensus review and statement regarding the anatomy, terminology, and rationale for lateral neck dissection in differentiated thyroid cancer. Thyroid 2012, 22:501-508.

10. Florentine BD, Staymates B, Rabadi M, Barstis J, Black A: Cancer Committee of the Henry Mayo Newhall Memorial Hospital: The reliability of fineneedle aspiration biopsy as the initial diagnostic procedure for palpable masses: a 4-year experience of 730 patients from a community hospitalbased outpatient aspiration biopsy clinic. Cancer 2006, 107:406-416.

11. Cignarelli M, Triggiani V, Ciampolillo A, Ambrosi A, Giorgino F, Liso V Giorgino R: High frequency of incidental diagnosis of extrathyroidal neoplastic diseases at the fine-needle aspiration biopsy of laterocervical lymph nodes in patients with thyroid nodules. Thyroid 2001, 11:65-71.

12. Ustün M, Risberg B, Davidson B, Berner A: Cystic change in metastatic lymph nodes: A common diagnostic pitfall in fine-needle aspiration cytology. Diagn Cytopathol 2002, 27:387-392.

13. Kessler A, Rappaport Y, Blank A, Marmor S, Weiss J, Graif M: Cystic appearance of cervical lymph nodes is characteristic of metastatic papillary thyroid carcinoma. J Clin Ultrasound 2003, 1:21-25.

14. Cignarelli M, Ambrosi A, Marino A, Lamacchia O, Campo M. Picca G, Giorgino F: Diagnostic utility of thyroglobulin detection in fine-needle aspiration of cervical cystic metastatic lymph nodes from papillary thyroid cancer with negative cytology. Thyroid 2003, 13:1163-1167.

15. Borel AL, Boizel R, Faure P, Barbe G, Boutonnat J, Sturm N, Seigneurin D, Bricault I, Caravel JP, Chaffanjon P, Chabre O: Significance of low levels of thyroglobulin in fine needle aspirates from cervical lymph nodes of patients with a history of differentiated thyroid cancer. Eur J Endocrinol 2008, 158:691-698.

16. Snozek CL, Chambers EP, Reading CC, Sebo TJ, Sistrunk JW, Singh RJ, Grebe SK: Serum thyroglobulin, high-resolution ultrasound, and lymph node thyroglobulin in diagnosis of differentiated thyroid carcinoma nodal metastases. J Clin Endocrinol Metab 2007, 92:4278-4281.

17 Salmaslıoğlu A, Erbil Y Cıtlak G, Ersöz F, Sarı S, Olmez A, Tunacı M, YIlmazbayhan D, Colak N, Ozarmağan S: Diagnostic value of thyroglobulin measurement in fine-needle aspiration biopsy for detecting metastatic lymph nodes in patients with papillary thyroid carcinoma. Langenbecks Arch Surg 2011, 396:77-81.

18. Kim MJ, Kim EK, Kim BM, Kwak JY, Lee EJ, Park CS, Cheong WY, Nam KH: Thyroglobulin measurement in fine-needle aspirate washout: the criteria for neck node dissection for patients with thyroid cancer. Clin Endocrinol 2009, 70:145-151.

19. Pacini F, Fugazzola L, Lippi F, Ceccarelli C, Centoni R, Miccoli P, Elisei R, Pinchera A: Detection of thyroglobulin in fine needle aspirates of nonthyroidal neck masses: a clue to the diagnosis of metastatic differentiated thyroid cancer. J Clin Endocrinol Metab 1992, 74:1401-1404.

20. Baskin HJ: Detection of recurrent papillary thyroid carcinoma by thyroglobulin assessment in the needle washout after fine-needle aspiration of suspicious lymph nodes. Thyroid 2004, 14:959-963.

21. Cunha N, Rodrigues F, Curado F, llhéu O, Cruz C, Naidenov P, Rascão MJ, Ganho J, Gomes I, Pereira H, Real O, Fiqueiredo P, Campos B, Valido F: Thyroglobulin detection in fine-needle aspirates of cervical lymph node: a technique for the diagnosis of metastatic differentiated thyroid cancer. Eur J Endocrinol 2007, 157:101-107.

22. Bournaud C, Charrié A, Nozières C, Chikh K, Lapras V, Denier ML, Paulin C, Decaussin-Petrucci M, Peix JL, Lifante JC, Cornu C, Giraud C, Orgiazzi J, Borson-Chazot F: Thyroglobulin measurement in fine-needle aspirates of lymph nodes in patients with differentiated thyroid cancer: a simple definition of the threshold value, with emphasis on potential pitfalls of the method. Clin Chem Lab Med 2010, 48:1171-1177.

23. Lee YH, Seo HS, Suh SI, Lee NJ, Kim JH, Seol HY, Lee JH, Kwon SY, Kim NH, Seo JA, Yang KS: Cut-off value for needle washout thyroglobulin in athyrotropic patients. Laryngoscope 2010, 120:1120-1124.

24. Frasoldati A, Toschi E, Zini M, Flora M, Caroggio A, Dotti C, Valcavi R: Role of thyroglobulin measurement in fine-needle aspiration biopsies of cervical lymph nodes in patients with differentiated thyroid cancer. Thyroid 1999, 9:105-111.

25. Sohn YM, Kim MJ, Kim EK, Kwak JY: Diagnostic performance of thyroglobulin value in indeterminate range in fine needle aspiration washout fluid from lymph nodes of thyroid cancer. Yonsei Med J 2012 53:126-131. 
26. Giovanella L, Cerlani L, Suriano S, Crippa S: Thyroglobulin measurement on fine-needle washout fluids: influence of sample collection methods. Diagn Cytopathol 2009, 37:42-44.

27. Kim DW, Jeon SJ, Kim CG: Usefulness of thyroglobulin measurement in needle-washout of fine-needle aspiration biopsy for the diagnosis of cervical lymph node metastases from papillary thyroid cancer before thyroidectomy. Endocrine 2012, 42:399-403.

28. Pomorski L, Kaczka K, Piaskowski S, Wójcik I, Rieske P, Matejkowska M, Kuzdak K: Detection of lymph node metastases of papillary thyroid cancer - comparison of the results of histopathology, immunohistochemistry and reverse transcription-polymerase chain reaction - a preliminary report. Langenbecks Arch Surg 2005, 390:209-215.

29. Basciani S, Watanabe M, Mariani S, Passeri M, Persichetti A, Fiore D, Scotto D'Abusco A, Caprio M, Lenzi A, Fabbri A, Gnessi L: Hypogonadism in a patient with two novel mutations of the luteinizing hormone betasubunit gene expressed in a compound heterozygous form. I Clin Endocrinol Metab 2012, 97:3031-3038.

30. Morgenthaler NG, Froehlich J, Rendl J, Willnich M, Alonso C, Bergmann A, Reiners $C$ : Techincal evaluation of a new immunoradiometric and a new immunoluminometric assay for thyroglobulin. Clin Chem 2002, 48:1077-1083.

31. Boi F, Baghino G, Atzeni F, Lai ML, Faa G, Mariotti S: The diagnostic value for differentiated thyroid carcinoma metastases of thyroglobulin (Tg) measurement in washout fluid from fine-needle aspiration biopsy of neck lymph node is maintained in presence of circulating anti-Tg antibodies. J Clin Endocrinol Metab 2006, 91:1364-1369.

32. Cianfarani F, Baldini E, Cavalli A, Marchioni E, Lembo L, Teson M, Persechino S, Zambruno G, Ulisse S, Odorisio T, D'Armiento M: TSH receptor and thyroid specific genes expression in human skin. J Invest Dermatol 2010, 130:93-101.

33. Baldini E, Sorrenti S, Catania A, Guaitoli E, Prinzi N, Mocini R, Nardi F, D'Armiento E, Bianchini M, Favoriti P, Di Matteo FM, Ruggieri M, De Antoni E, Ulisse S: Diagnostic utility of thyroglobulin measurement in the fine needle aspirates from cervical lymph nodes: a case report. G Chir 2012, 33:387-391

doi:10.1186/1472-6890-13-7

Cite this article as: Baldini et al:: Cervical lymph node metastases from thyroid cancer: does thyroglobulin and calcitonin measurement in fine needle aspirates improve the diagnostic value of cytology?. BMC Clinical Pathology 2013 13:7.

\section{Submit your next manuscript to BioMed Central and take full advantage of:}

- Convenient online submission

- Thorough peer review

- No space constraints or color figure charges

- Immediate publication on acceptance

- Inclusion in PubMed, CAS, Scopus and Google Scholar

- Research which is freely available for redistribution 